\title{
Antibody to the gastric campylobacter-like organism ("Campylobacter pyloridis") —clinical correlations and distribution in the normal population
}

\author{
D. M. JONES, J. ELDRIDGE, A. J. FOX, P. SETHI* AND P. J. WHORWELL†
}

\begin{abstract}
Public Health Laboratory, and Departments of *Geriatric Medicine and TMedicine, Withington Hospital, Manchester M20 8LR
\end{abstract}

\begin{abstract}
Summary. Different cellular proteins of the gastric campylobacter-like organism (GCLO) were shown to be immunogenic for man. Antibodies to GCLO were detected in sera by both complement fixation and enzyme-linked immunoabsorbent assay. Antibody was found in $133(52 \%)$ of 254 patients attending for gastroscopy. There was a high correlation between presence of antibody and a positive GCLO culture from the gastric mucus. Patients with normal endoscopic appearances, duodenal ulcer, duodenitis and oesophagitis had similar prevalences $(c .50 \%)$ of antibody. Only patients with endoscopically visible gastritis or gastric ulcer had a higher frequency $(c$. $80 \%$ ) of antibody. In a normal population, antibody was uncommon in individuals $<20$ years old, but the prevalence of antibody increased (to $c .50 \%$ ) with age. There was little evidence to support an important pathological role for GCLO in disorders of the upper gastrointestinal tract, although the possibility that it may be a co-factor in the pathogenesis of gastric ulcer cannot be excluded.
\end{abstract}

\section{Introduction}

In a previous study of 50 unselected patients attending for gastroscopy, we established a close association between the presence of the gastric campylobacter-like organism (GCLO, "Campylobacter pyloridis") in the gastric mucus and the presence of histologically demonstrable gastritis (Jones et al., 1984). This association has been observed by others (Langenberg et al., 1984, Marshall and Warren, 1984; McNulty and Watson, 1984) but the degree of association with other forms of upper gastrointestinal pathology remains obscure, although it has been suggested that these organisms may at least be a co-factor in the pathogenesis of peptic ulceration (Marshall et al., 1985). Many studies are in progress to assess the effects of anti-ulcer therapy and antibiotics on these organisms in an effort to elucidate any causal relationship with peptic ulceration.

In our earlier study we also established a close association between circulating antibody to GCLO and the presence of GCLO in the stomach, and observed a similar close association between antibody and histological gastritis. We have extended the study to a larger number of unselected patients undergoing endoscopy to obtain more substantial

Received 31 Oct. 1985; accepted 29 Nov. 1985. numbers with different endoscopic findings and report the prevalence of antibody in such patients. We have also studied selected patients whose endoscopic appearances were normal and report the culture, serological and histological findings. As antibody estimation offers a way of studying the distribution of the organism in a more general population, we have made a serological survey of antibody in groups of individuals of all ages with a view to placing the results obtained with endoscopic patients in a more general epidemiological context.

\section{Materials and methods}

\section{Patients}

One-hundred and ninety unselected patients referred for gastroscopy were studied. In addition to the routine histological examination of the biopsies taken, many were cultured for GCLO and blood samples were collected for measurement of antibody to GCLO. A further 64 patients whose endoscopic appearances were normal were studied. In each patient, biopsy specimens were obtained from the gastric antrum and greater curve and blood was taken for antibody estimation. The indications for endoscopy were symptoms referable to the upper gastrointestinal tract. For comparison, sera that had been collected from 607 individuals of all ages were examined for antibody to GCLO. These individuals were patients 
without gastrointestinal conditions, healthy individuals from health-screening clinics and other healthy normal children and aduits.

\section{Methods}

Fresh gastric-biopsy specimens were examined by direct microscopy of gram-stained mucus and by culture on blood agar as previously described (Jones et al., 1984). Biopsy specimens were also fixed and stained and the histological appearances classified as normal, as chronic superficial gastritis or as atrophic gastritis according to the criteria suggested by Whitehead (1979).

\section{Serology}

Antibody was estimated by two methods. Complement-fixation (CF) tests were performed with a sonicate of six GCLO isolates as antigen (Jones et al.. 1984). Most sera were also tested in parallel by enzyme-linked immunoabsorbent assay (ELISA) for the presence of IgG- and IgM-class antibodies with a whole-cell antigen. A suspension of live organisms of six different GCLO strains $\left(c .10^{8}\right.$ organisms $/ \mathrm{ml}$ ) was diluted 1 in 20 in carbonate-bicarbonate buffer, $p \mathrm{H}$ 9.6. Plastic, 96-well microtitration plates (NUNC) were coated overnight at $4 \mathrm{C}$ and then washed three times in water. Plates were used within $48 \mathrm{~h}$ of preparation. The horse-radish peroxidase system was used with $\mathrm{H}_{2} \mathrm{O}_{2}-O$-phenylenediamine as substrate (Voller et al. 1979). All sera from endoscopy patients were titrated. In the survey of normal individuals, sera were screened at a dilution of 1 in 300 by ELISA and titrated in the CF test.

\section{Immunoblotting}

Suspensions of six isolates of GCLO were extracted with EDTA-lysozyme buffer (Mills and Bradbury, 1984). The extracts were run on $10 \%$ polyacrylamide gels. After SDS-PAGE fractionation, the separated components were transferred to nitrocellulose sheets (Biorad) (Towbin et al., 1979). Serum diluted 1 in 50 in Tris- $\mathrm{NaCl}$ buffer with Tween $200.05 \%$ was left in contact with the nitrocellulose for $2 \mathrm{~h}$ at room temperature. The sheets were then washed in buffer and anti-human alkalinephosphatase conjugate applied. After a final wash antibody binding was detected with a colour-development agent (Acid-fast red TR salt, naphthol ASMX phosphoric acid; Sigma).

\section{Results}

\section{Immunoblotting}

Six strains of GCLO gave very similar polypeptide profiles when examined by SDS-PAGE; these were quite different from the profiles of $C$. jejuni (Fig. 1). From examination of the immunoblots of sera from patients with antibody detectable by $\mathrm{CF}$ or ELISA, there appeared to be major reactions with antigens of mol. wts $\left(10^{3}\right) 14-21$ and 33 (Fig. 2) from all GCLO strains and these antigens were

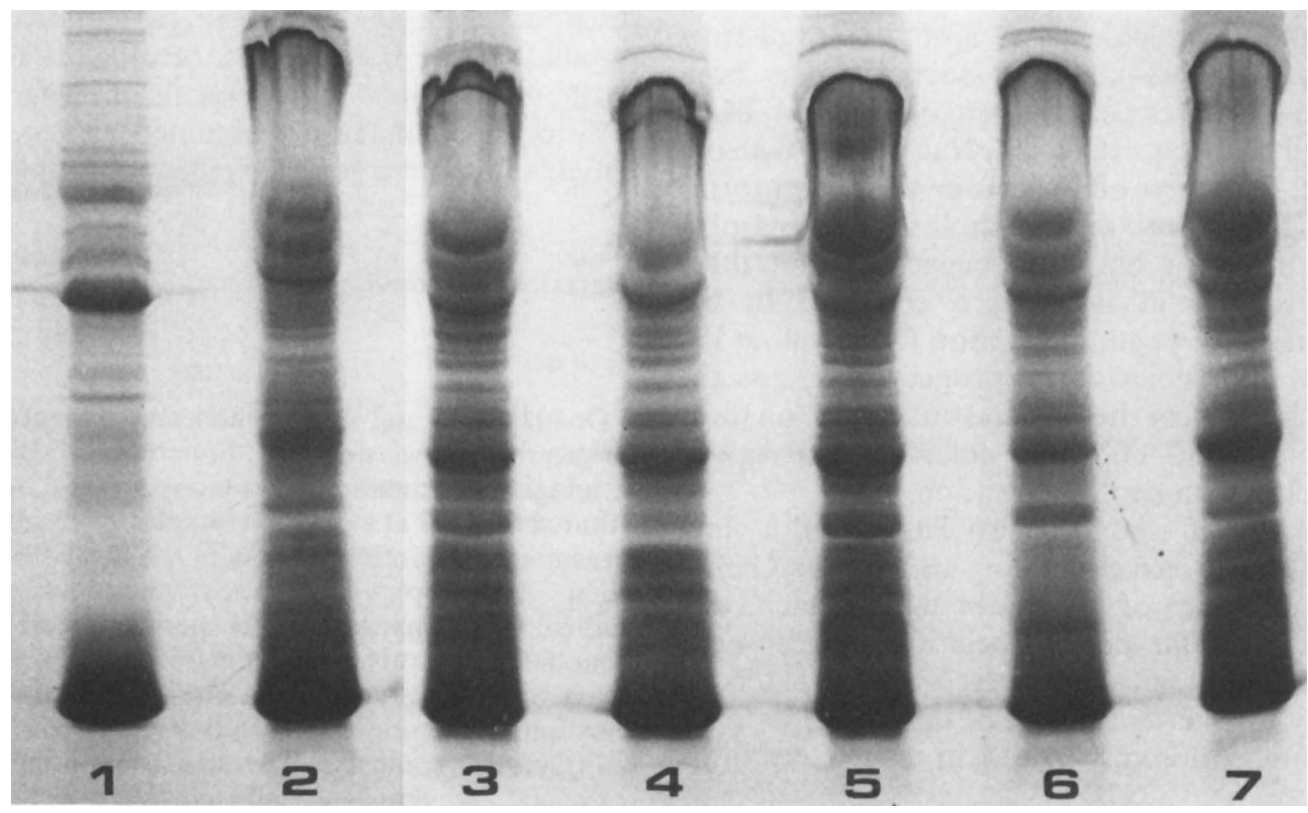

Fig. 1. Polyacrylamide-gel electrophoresis of extracts of C. jejuni (track 1) and strains of GCLO (tracks $2 \cdots 7$ ). 


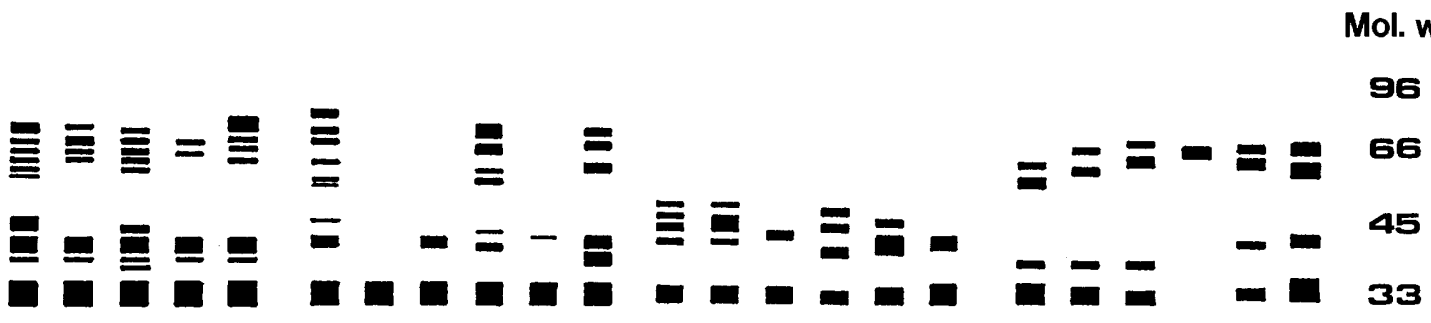

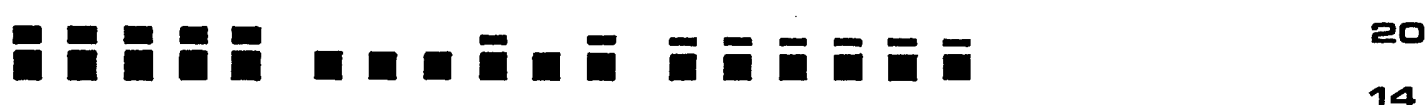 \\ $\frac{12345}{A} \frac{123456}{B} \frac{123456}{1}$}

Fig. 2. Immunoblots of antibody-containing sera with strains of GCLO. Serum from patient: (A) shows the usual pattern of response; (B) did not react with high mol.-wt bands from some strains; (C) did not react with high mol.-wt bands from any strain; (D) did not react with low mol.-wt bands from any strain.

similar in all strains examined. Sera also reacted with antigens of mol. wts $\left(10^{3}\right) 55-70$ but this reactivity was not found with all strains, indicating both variation in the antibody response to these antigens and variation in the antigens themselves. No significant differences were observed between immunoblots of healthy people with antibody and endoscopy patients with demonstrable gastritis and antibody. Immunoblots prepared from antibodynegative sera showed no evidence of reaction with GCLO antigens; these same sera reacted with a number of antigens of $C$. jejuni, a regular finding in sera with antibody to $C$. jejuni undetectable by conventional methods.

\section{Serological tests}

The CF test gave titres in the range 2-64 and the ELISA titres were in the range 300-2400. Titres $<300$ by ELISA were regarded as non-specific. The results of the two methods were in close accord although the ELISA was more sensitive than complement fixation. By ELISA all antibody-positive sera contained IgG antibody; about $10 \%$ of sera examined had IgM antibody but this could not be correlated with any particular condition. Examination of a small number of stored sera showed that antibody to GCLO may remain at the same level in individuals over periods of at least 8-12 years and possibly longer.

\section{Endoscopically normal patients}

GCLO were grown from 31, and antibody was demonstrated in 30 , of the 64 endoscopically normal patients. Histological examination of the 64 biopsies demonstrated chronic superficial gastritis in 26 patients $(41 \%), 17$ of whom were $<50$ years old and 9 were $>70$ years old. Atrophic gastritis was present in 17 patients $(27 \%)$, all of whom were

Table I. Histological appearance, culture and antibody results from biopsies of 64 endoscopically normal patients

\begin{tabular}{cccc}
\hline & & Number $(\%)$ positive for GCLO by \\
\cline { 3 - 4 } $\begin{array}{c}\text { Histological } \\
\text { appearance* }\end{array}$ & $\begin{array}{c}\text { Number of } \\
\text { patients }\end{array}$ & culture & serology \\
\hline CSG & 26 & $21(80)$ & $18(69)$ \\
AG & 17 & $9(53)$ & $12(76)$ \\
N & 21 & $1(5)$ & 0 \\
\hline
\end{tabular}

${ }^{*} \mathrm{CSG}=$ chronic superficial gastritis; $\mathrm{AG}=$ atrophic gastritis; $\mathrm{N}=$ normal. 
$>70$ years old. The remaining 21 patients $(33 \%)$ had no histological abnormality. The relationship between histological appearance, culture and presence of antibody is shown in table I. Whereas patients with either chronic superficial gastritis or atrophic gastritis had a similar prevalence of antibody, a positive culture was less frequent from biopsies showing mucosal atrophy, possibly because less mucus was present on the mucosa. As in our previous study, a positive GCLO culture or antibody result was not common in the presence of a histologically normal mucosa. The association between histological gastritis and antibody did not differ significantly with age, although an atrophic mucosa was more likely in the elderly. Biopsies showing an atrophic mucosa could be divided into those with some inflammatory response and those from which this was absent; antibody to GCLO was found to be present only in those patients who had an inflammatory response in the gastric mucosa.

\section{Unselected endoscopy-clinic patients}

The relationship between endoscopic appearances and the presence of antibody to GCLO in 190 patients is shown in table II. About half of the patients with duodenitis, duodenal ulcer, oesophagitis, or whose endoscopic appearances were normal had antibody to GCLO. Only patients with gastric ulcer and visible gastritis had a higher prevalence of antibody. The mean antibody titres were the same in all groups except for the patients with visible gastritis in whom it was higher, possibly signifying a more active inflammatory process in these patients.

\section{Population survey}

The results of testing 607 sera from individuals

Table II. Presence of antibody to GCLO in 190 patients attending for gastroscopy

\begin{tabular}{lcc}
\multicolumn{1}{c}{ Diagnosis } & $\begin{array}{c}\text { Number of } \\
\text { patients }\end{array}$ & $\begin{array}{c}\text { Number }(\%) \text { with } \\
\text { GCLO antibody }\end{array}$ \\
\hline Gastric ulcer & 14 & $12(86)$ \\
Gastritis & 18 & $14(77)$ \\
Duodenal ulcer & 29 & $17(59)$ \\
Oesophagitis & 43 & $16(37)$ \\
Duodenitis & 21 & $9(43)$ \\
Tumour & 3 & $2(66)$ \\
Oesophageal varices & 1 & 0 \\
Normal & 61 & $32(52)$ \\
\hline
\end{tabular}

Table III. The distribution, by age, of antibody to GCLO in sera from 607 control individuals

\begin{tabular}{ccc}
$\begin{array}{c}\text { Age } \\
\text { (years) }\end{array}$ & $\begin{array}{c}\text { Number of } \\
\text { individuals }\end{array}$ & $\begin{array}{c}\text { Number }(\%) \text { with } \\
\text { GCLO antibody }\end{array}$ \\
\hline $6-9$ & 22 & $1(5)$ \\
$10-14$ & 87 & 0 \\
$15-20$ & 151 & $9(6)$ \\
$21-30$ & 64 & $12(19)$ \\
$31-40$ & 53 & $10(19)$ \\
$41-50$ & 37 & $6(16)$ \\
$51-60$ & 28 & $11(40)$ \\
$61-70$ & 43 & $21(49)$ \\
$71-80$ & 69 & $34(49)$ \\
$81-90$ & 53 & $22(41)$ \\
\hline
\end{tabular}

aged 6 years and upwards are shown in table III. Antibody was found in few children and then the incidence gradually increased with age but did not rise above the $50 \%$ level even in the elderly.

\section{Discussion}

When examined by SDS-PAGE the protein profiles of six strains of GCLO were very similar and quite different from those of $C$. jejuni, in agreement with the observations of Pearson et al (1984). Immunoblotting with antibody-containing sera demonstrated that many of the protein bands from the GCLO were immunogenic for man. Reactions were always found with the major protein bands of mol. wts $\left(10^{3}\right) 14-21$ and 33. Proteins of greater mol. wt., probably associated with flagellar antigens, were not reactive with all sera, suggesting some degree of antigenic variation among strains and variations of immunological response in different subjects. However, the regular reactions with the major protein bands suggested that the antigens used for the serological tests would be likely to detect antibody, when present, in most, if not all, sera. When antibody-negative sera were blotted, there were no reactions with proteins of GCLO but there were reactions with antigens of $C$. jejuni. That antibody-negative normal sera react with antigens derived from $C$. jejuni has been observed before (Blaser et al., 1984), suggesting that for that organism experience with some of its antigens, or others very closely related, is an almost universal experience. In this respect, the GCLO is quite different; the negative sera gave no traces of reaction, which suggests that infection with this organism is not often experienced in man. The continued presence 
of antibody in samples collected over many years from the same person is also in keeping with the view that individuals are infected or colonised or neither and that there may be a stable long-lasting relationship with the host.

For this serological study, we used two techniques with two different antigen preparations. The very close agreement between the ELISA and CF results gave us confidence in the specificity of the tests. Some cross-reactions occurred in sera from patients with high antibody titres to $C$. jejuni when examined by ELISA, but these were not apparent with the rather less sensitive CF test. It is, however, our practice always to test for antibody to $C$. jejuni when testing for antibody to GCLO.

In this and our earlier study, the close association of three factors - the presence of the organism in the gastric mucus, histological gastritis, and antibody-was clearly evident. The selection of patients who were endoscopically normal puts the findings into a frame uncomplicated by the presence of gastric or duodenal pathology. The organism and antibody were present in about half of the 64 patients in that group and in all of those with antibody who had either chronic superficial gastritis or atrophic gastritis. Twelve of the patients had gastritis without the presence of organism or antibody; thus, c. $75 \%$ of patients with gastritis appeared to have evidence of GCLO infection. Rather than assign a causal relationship between GCLO and the inflammatory response, this finding may be taken as evidence that the presence of inflammation in the mucosa is a necessary prerequisite for colonisation by GCLO. It is perhaps only in the presence of some tissue damage that the necessary metabolites for growth of the organism are released from the gastric mucosa, thus encouraging colonisation of the potentially hostile environment of the gastric mucus. An alternative is that inflammation leads to an alteration in the character of the mucus that in turn favours colonisation with GCLO. Although the number of patients was rather small, it was interesting to observe that atrophy of the gastric mucosa per se was unrelated to the presence of the organism or antibody, which was correlated in particular with the presence of an inflammatory response in the mucosa. Again, there was quite clear evidence from this study that a histologically normal mucosa (as opposed to endoscopically normal appearances) in patients was not usually associated with the presence of GCLO or its antibody.

In the unselected patients attending for gastroscopy, the prevalence of antibody in patients in the various clinical groups made an interesting com- parison. Of the patients from this series with duodenal ulcers, duodenitis, oesophagitis or with normal endoscopic appearances, about half had antibody to GCLO and, thus, by implication were colonised or infected with GCLO. The inference is that the role of GCLO in all these conditions may be the same and that patients with these diverse conditions have the same rate of colonisation or infection. Furthermore, the findings did not differ significantly from those in the selected group of endoscopically normal patients. GCLO is, therefore, unlikely to contribute anything more than a histologically demonstrable inflammatory response and the results lead to the supposition that an antibody rate of $c .50 \%$ may be a normal finding in adults. Of the patients studied, only those with obvious, visible gastritis or gastric ulcers had a higher prevalence of antibody of $c .80 \%$. This raises the possibility that GCLO have a more active role in the pathogenesis of these conditions.

From examination of the sera collected from normal people, it is clear that antibody is uncommon in children and teenagers. The prevalence of antibody increases to $c .50 \%$ with age suggesting that the organism is acquired during life. This poses the question of how, when and from what source the organism is acquired. It appears to be adapted for growth in gastric mucus and its isolation has not been reported from any site other than the proximity to a gastric-type mucosa. Isolation methods are still relatively insensitive so that it remains conjectural whether small numbers of this organism will be found on other mucosal surfaces of the body. Persistence of antibody in an individual, together with the complete absence of reactions with antibody-negative sera by immunoblotting, suggest that antibody and, therefore, the organism may not be ephemeral and that, once acquired, it inhabits the gastric mucus for very long periods. The finding that adults without gastrointestinal symptoms have a very similar overall prevalence of antibody to GCLO as those attending for gastroscopy tends to detract from the hypothesis that the organism has a definitive role in the pathophysiology of upper gastrointestinal diseases. The association between GCLO and histologically demonstrable gastritis is unequivocal, although whether cause or effect remains to be determined. However, this study fails to support a substantial pathogenic role for GCLO in other upper gastrointestinal disorders, although the possibility that it acts as a co-factor in gastric ulceration cannot be excluded.

We are grateful for the collaboration of many colleagues, in particular Drs P. Miller and A. K. Banerjee. 


\section{REFERENCES}

Blaser M J. Hopkins J A, Vasil M L 1984 Campilobacter jejuni outer membrane proteins are antigenic for humans. Infe'clion and Immunity 43:986-993.

Jones D M. Lessells A M. Eldridge J 1984 Campylobacter-like organisms on the gastric mucosa: culture, histological and serological studies. Journal of Clinical Patholog! 37:1002. 1006.

Langenberg M-L. Tytgat G N J. Schipper M E I. Rietra P J G M. Zanen HC 1984 Campylobacter-like organisms in the stomach of patients and healthy individuals. Lancet 1:1348.

Marshall B J. Warren J R 1984 Unidentified curved bacilli in the stomach of patients with gastritis and peptic ulceration. Lumc'l 1:1311-1314.

Marshall B J. Armstrong J A. McGechie D B. Glancy R J 1985 Attempt to fulfil Koch's postulates for pyloric campylohacter. Modical Journal of tustralia 142:436 439.
Mills S D. Bradbury W C 1984 Human antibody response to outer membrane proteins of Campylobacter jejuni during infection. Infection and Immunity 43: 739--743.

McNulty C A M, Watson D M 1984 Spiral bacteria of the gastric antrum. Lancet 1:1068-1069.

Pearson A D. Bamforth J. Booth L. Holdstock G, Ireland A. Walker C. Hawtin P. Millward-Sadler H 1984 Polyacrylamide gel electrophoresis of spiral bacteria from the gastric antrum. Lancet 1:1349-1350.

Towbin H. Staehelin T, Gordon J 1979 Electrophoretic transfer of proteins from polyacrylamide gels to nitrocellulose sheets: procedure and some applications. Proceedings of the Vational Academi of Sciences of the United States of America 76:4350 4354.

Voller A. Bidwell D E, Bartlett A 1979 The enzyme linked immunosorbent assay. Dynatech Europe. pp 18-40.

Whitehead R 1979 Mucosal biopsy of the gastro-intestinal tract, Ind edn. W B Saunders. London, pp 2032. 